\title{
Economical Benefits of Energy Recovery from the Undesirable Components of Electric Signals
}

\author{
Moisés V. Ribeiro, Victor Fernandes, Nathan Cravo, Henrique L. M. Monteiro
}

\begin{abstract}
This paper introduces a practical approach to analyze and quantify the benefits of using the waste-to-energy concept to improve power quality and energy efficiency in electric power systems. Also, we introduce the so-called Passive Filtering Energy Recovery, which aims to perform the energy recovery in undesirable components present in electric signals. Finally, numerical results show that this approach can improve power quality and energy efficiency in the Brazilian scenario.
\end{abstract}

Keywords - waste-to-energy, energy recovery, power quality, energy efficiency, electric power systems.

\section{INTRODUCTION}

It is notorious in today's society the growing electricity dependency, which is been leveraging an increasing evolution of electric power systems. With this perspective, an accurate and on-demand use of energy deserves attention. In this sense, quantifying and analyzing how well energy is been consumed is an important factor, and this where Waste-to-Energy (WtE) [1] comes to. It is an approach that can be used to estimate and quantify energy losses due to various energy production processes, such as wind, solar and thermal energy production. Also, it allows us to broaden our vision regarding energy losses in electric power systems. In this regard, this paper proposes to apply WtE to energy recovery in electric power systems, considering that minimizing such losses can positively impact energy efficiency and power quality with respect to generation, transmission, distribution, and electricity consumption. Concerns about wasted energy in electric power systems is a recurrent issue in literature [2], and an accurate evaluation of technical and non-technical losses in this systems exert noteworthy influence on technical, economic, and regulatory issues [3], as will be presented later in this paper as well.

With this in mind, the wasted energy accounted by us is the one that arises because of the interaction between nonlinear loads and electric power systems, and will be called undesirable components from now on. Such components appear because part of the supplying energy, generated by energy sources and delivered to consumers through the fundamental

This study was supported in part by Coordenação de Aperfeiçoamento de Pessoal de Nível Superior (CAPES) - finance code 001, in part by Conselho Nacional de Desenvolvimento Científico e Tecnológico $(\mathrm{CNPq})$, in part by Instituto Nacional de Energia Elétrica (INERGE), and in part by Fundação de Amparo à Pesquisa do Estado de Minas Gerais (FAPEMIG).

Moisés V. Ribeiro, Victor Fernandes, and Nathan Cravo are with Electrical Engineering Department, Federal University of Juiz de Fora, Juiz de Fora, MG 36036-900 Brazil, (e-mails: \{mribeiro, victor.fernandes, nathan.cravo \}@engenharia.ufjf.br)

Henrique L. M. Monteiro is with Institute of Science, Technology and Innovation of São Sebastião do Paraíso, Federal University of Lavras, Lavras, MG 37200900 Brazil, (e-mail: henrique.monteiro@ufla.br). frequency, is wasted due to its spread over the spectrum as waveform distortions (e.g., Direct Current (DC), harmonics, interharmonics, supraharmonics, and transients), which happens regardless of thermal losses, being these losses not approached in our analysis. Additionally, the existing energy in undesirable components is also a source of Power Quality (PQ) degradation and energy inefficiency in electric power systems, being a motivation for searching the underlying causes of such wasted energy in electric signals and the design of appropriate countermeasures to mitigate them, which has been addressed in the literature before [4].

These components are commonly disregarded by the electricity sector; however, they impose considerable PQ degradation in electric power systems. Hence, research efforts aiming to address the wasted energy associated with undesirable components in electric signals are necessary, considering that such components emerge naturally from the interaction between nonlinear loads and the electric power systems.

With this perspective, an emerging research direction is to assume that these undesirable components are additional sources of energy because the wasted energy associated with them can be reused after an appropriate energy recovery process, as initially approached in [5, 6]. The first one addressed energy recovery in chip while [6] focused on Power Line Communication (PLC) additive noise. An interesting point is that both took advantage of the $\mathrm{WtE}$ concept: exploit the hidden or neglected energy in disregarded components. In electric power systems, the energy recovery process can result in PQ and energy efficiency improvements. Thus, we highlight the importance of quantifying the wasted energy in undesirable components in electric signals and the deployment of energy recovery technologies for this purpose.

In this sense, this paper aims at addressing the benefits of recovering the wasted energy in such undesirable components and advancing the energy losses discussion in electric power systems beyond the fundamental and harmonic components. To do so, we introduced the so-called Passive Filtering Energy Recovery (PFER) [7] to foster the design of power-electronicbased devices that are capable of performing energy recovery and, as a consequence, increasing PQ and energy efficiency in electric power systems. Also, based on a measured data set we present a numerical analyses to illustrate technical and economic improvements that can be attained by using a PFER for performing the energy recovery in undesirable components.

\section{Problem Formulation}

In our problem formulation we consider an electric power system without thermal and corona losses. These are disre- 


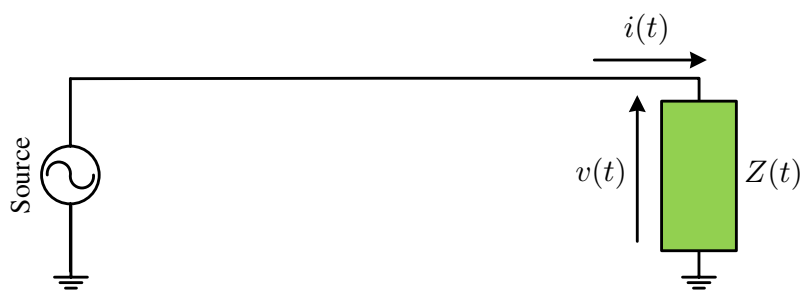

Fig. 1: The single-phase model composed of source, ideal transmission line, and nonlinear load.

garded because they are not of interest to the discussion of using waste-to-energy in undesirable components in electric signals. Also, let this system be comprised of an Alternating Current (AC) energy source (fundamental frequency equal to 50 or $60 \mathrm{~Hz}$ ), a lossless transmission line, and a nonlinear load $^{1}$, as shown in Figure 1. The AC energy source generates a complex power equal to $N_{\text {signal }}=P_{\text {signal }}+j Q_{\text {signal }}$, and $Z(t)$ denotes the dynamic of this nonlinear load. The presence of a DC energy source is omitted in Figure 1 since it is tiny in comparison to the AC energy source. The assumption of an isolated electric power system offers a sufficient condition for applying the first law of thermodynamics (i.e., the law of energy conservation) in this model, which is used to justify the energy recovery scheme.

Due to the nonlinearity of the loads, the voltage signal at the terminals of this nonlinear load, after interacting with the electric power system, is given by [8]

$$
\begin{aligned}
v(t) & =V_{0}+\sum_{k=1}^{N_{1}} V_{k}(t) \cos \left(k \omega_{1} t+\theta_{v, k}(t)\right)+ \\
& \sum_{l=1}^{N_{2}} V_{I, l}(t) \cos \left(\omega_{I, l} t+\theta_{v, I, l}(t)\right)+ \\
& \sum_{l=1}^{N_{3}} V_{S, l}(t) \cos \left(\omega_{S, l} t+\theta_{v, S, l}(t)\right)+ \\
& \sum_{l=1}^{N_{4}} v_{\text {imp }, l}(t)+\sum_{l=1}^{N_{5}} v_{\text {not }, l}(t)+\sum_{l=1}^{N_{6}} v_{\text {osc }, l}(t)+v_{n}(t),
\end{aligned}
$$

where $-\infty<t<+\infty$; $V_{0}$ is the DC voltage component; $\left\{V_{1}(t), \Omega_{1}, \theta_{1}(t)\right\}, \quad\left\{V_{k}(t), \Omega_{k}, \theta_{k}(t)\right\}, \quad\left\{V_{I, l}(t), \Omega_{I, l}, \theta_{I, l}(t)\right\}$ and $\left\{V_{S, l}(t), \Omega_{S, l}, \theta_{S, l}(t)\right\}$ are parameters of time-varying fundamental, harmonic, interharmonic and supraharmonic components, respectively; $\Omega_{1}=2 \pi f_{1}$ and $f_{1}$ is the fundamental frequency. Also, $v_{\text {imp }, l}(t), v_{\text {not }, l}(t), v_{\mathrm{osc}, l}(t)$ denote the $l$ th impulse, the $l$ th notch and the $l$ th damped oscillatory transients, respectively, which are supposed to be random processes. Note that $N_{1}, N_{2}$ and $N_{3}$ are the number of fundamental plus harmonic, interharmonic, and supraharmonic components, respectively, while $N_{4}, N_{5}$ and $N_{6}$ refer to the number of the aforementioned transient components. Also, we assume that the additive noise, $v_{n}(t)$, and transient components are zero mean wide-sense stationary random process, and, as a consequence, $v(t)$ is a random process. Similar to (1) the

\footnotetext{
${ }^{1}$ Other sources of noise, such as radio signals induced in power lines, are not considered.
}

current signal is given by

$$
\begin{aligned}
& i(t)=I_{0}+\sum_{k=1}^{N_{1}} I_{k}(t) \cos \left(k \omega_{1} t+\theta_{i, k}(t)\right)+ \\
& \sum_{l=1}^{N_{2}} I_{I, l}(t) \cos \left(\omega_{I, l} t+\theta_{i, I, l}(t)\right)+ \\
& \sum_{l=1}^{N_{3}} I_{S, l}(t) \cos \left(\omega_{S, l} t+\theta_{i, S, l}(t)\right)+ \\
& \sum_{l=1}^{N_{4}} i_{\mathrm{imp}, l}(t)+\sum_{l=1}^{N_{5}} i_{\mathrm{not}, l}(t)+\sum_{l=1}^{N_{6}} i_{\mathrm{osc}, l}(t)+i_{n}(t),
\end{aligned}
$$

Also, the assumptions used for $v(t)$ apply to $i(t)$. Let us assume that $v(t) \in \mathbb{R}, i(t) \in \mathbb{R}, \mathbb{E}\{$.$\} is the expectation$ operator. and $\star$ the conjugation operation. Consequently, the analytic cross-power spectral density between $v(t)$ and $i(t)$ is defined by

$$
\begin{aligned}
S_{\mathrm{aVI}}(j \Omega) & \triangleq \int_{-\infty}^{+\infty} \mathbb{E}\left\{v_{\mathrm{a}}(t) i_{\mathrm{a}}^{*}(t-\tau)\right\} e^{-j \Omega \tau} d \tau, \\
& =\lim _{T \rightarrow \infty} \mathbb{E}\left\{\frac{V_{\mathrm{a} T}(j \Omega) I_{\mathrm{a} T}^{*}(j \Omega)}{T}\right\},
\end{aligned}
$$

where $v_{\mathrm{a}}(t)=v(t)+j \hat{v}(t)$ and $i_{\mathrm{a}}(t)=i(t)+j \hat{i}(t)$ are the analytic signal representation for (1) and (2), respectively, while $\hat{v}(t)$ and $\hat{i}(t)$ are the Hilbert transforms of (1) and (2), respectively.

The so-called Extended Complex Power (ECP) is obtained by evaluating the following expression:

$$
N_{E C}=\int_{0}^{+\infty} K(j \Omega) S_{\mathrm{aVI}}(j \Omega) d \Omega,
$$

where

$$
K(j \Omega)= \begin{cases}2, & \Omega=0, \\ \frac{1}{2}, & \Omega>0,\end{cases}
$$

$\Delta \theta_{k}=\theta_{v, k}-\theta_{i, k}, \Delta \theta_{I, l}=\theta_{v, I, l}-\theta_{i, I, l}, \Delta \theta_{S, l}=\theta_{v, S, l}-$ $\theta_{i, S, l}$. Also, $S_{\text {aimp }, l}(j \Omega), S_{\text {anot }, l}(j \Omega), S_{\text {aosc }, l}(j \Omega)$, and $S_{\text {an }}(j \Omega)$ are the analytic cross-power spectral densities for the transient and additive noise components, respectively. After some manipulations, we come up with the following expression for ECP:

$$
\begin{aligned}
N_{E C} & =P_{E C}+j Q_{E C} \\
& \triangleq \frac{V_{0} I_{0}}{2}+\sum_{k=1}^{N_{1}} \frac{V_{k} I_{k}}{2} e^{j \Delta \theta_{k}}+\sum_{l=1}^{N_{2}} \frac{V_{I, l} I_{I, l}}{2} e^{j \Delta \theta_{I, l}}+ \\
& \sum_{l=1}^{N_{3}} \frac{V_{S, l} I_{S, l}}{2} e^{j \Delta \theta_{S, l}}+\frac{1}{8 \pi^{2}}\left(\sum_{l=1}^{N_{4}} \int_{0}^{+\infty} S_{\text {aimp }, l}(j \Omega) d \Omega+\right. \\
& \sum_{l=1}^{N_{5}} \int_{0}^{+\infty} S_{\text {anot }, l}(j \Omega) d \Omega+\sum_{l=1}^{N_{6}} \int_{0}^{+\infty} S_{\text {aosc }, l}(j \Omega) d \Omega+ \\
& \left.\int_{0}^{+\infty} S_{\text {an }}(j \Omega) d \Omega\right) .
\end{aligned}
$$

Note that $N_{E C}$ is obtained considering the signals $v(t)$ and $i(t)$ at the terminal of a nonlinear load. This parameter can be 
used to estimate the wasted energy associated with undesirable components (e.g., harmonics, interharmonics, supraharmonics and transients). In other words, the complex power $N_{\text {trash }}$, which is directly obtained from (6) by not taking the fundamental frequency contribution. Note that this portion can be seen as the useful complex power called $N_{\text {useful }}$, a portion of the AC energy source delivered to the nonlinear load by using the fundamental frequency. Note that $N_{E C}$ reduces to the wellknown complex power, $N_{A}$ (apparent complex power), where both $v(t)$ and $i(t)$ are solely composed of fundamental and harmonic components. In other words, $N_{A}$ is a particular case of $N_{E C}$, and the corresponding complex power is as follows:

$$
\begin{aligned}
N_{A} & =N+N_{h} \\
& =P+j Q+P_{h}+j Q_{h} \\
& =\frac{V_{1} I_{1}}{2} e^{j \Delta \theta_{1}}+\sum_{k=2}^{N_{1}} \frac{V_{k} I_{k}}{2} e^{j \Delta \theta_{k}},
\end{aligned}
$$

$N=P+j Q=\frac{V_{1} I_{1}}{2} e^{j \Delta \theta_{1}}$ and $N_{h}=P_{h}+j Q_{h}=$ $\sum_{k=2}^{N_{1}} \frac{V_{k} I_{k}}{2} e^{j \Delta \theta_{k}}$

Giving the assumption of an isolated system and the models of voltage and current signals, see (1) and (2), it is seen that the energy of harmonics, interharmonics, supraharmonics, transients, and additive noise come from the energy delivered to the load by the AC energy source. In the electricity sector, such components are considered waste that degrades PQ and energy efficiency, and consequently, a great deal of attention is toward their mitigation. Also, the usefulness of the wasted energy within such components is noteworthy because it can be useful to supply low-power consumption loads (e.g., Internet of Things (IoT) devices).

Grounded on the $\mathrm{WtE}$ concept the next sections present how beneficial is to recover the wasted energy in undesirable components and illustrate how the so-called Passive Filtering Energy Recovery can be used in this process.

\section{PASSIVE FILTERING ENERGy RECOVERY}

Passive Filtering is a technique in which passive elements such as resistors, capacitors and inductors are used. This filtering can be defined to mitigate the current harmonic content. With this, the value of each component is defined according to the harmonic frequency mitigated in the system. In this sense, Fig. 2 shows the Passive Filtering element applied in a electric power system. As can be seen, the filter is connected in parallel with the nonlinear load and the energy source. The energy absorbed by the filter is the $N_{\text {wasted }}+N_{\text {noise }}$.

However, no devices make use of the harmonic energy mitigated solely by this approach. Thus, the Energy Recovery becomes promising since the energy will be stored and utilized by low-energy devices. With this in mind, our goal is to insert a device in series with the Passive Filtering to consume the harmonic energy absorbed by the filtering process. Hence, Fig. 3 illustrates the circuit with a low-energy device added in series with the Passive Filtering. The device is defined as Passive Filtering Energy Recovery device, an

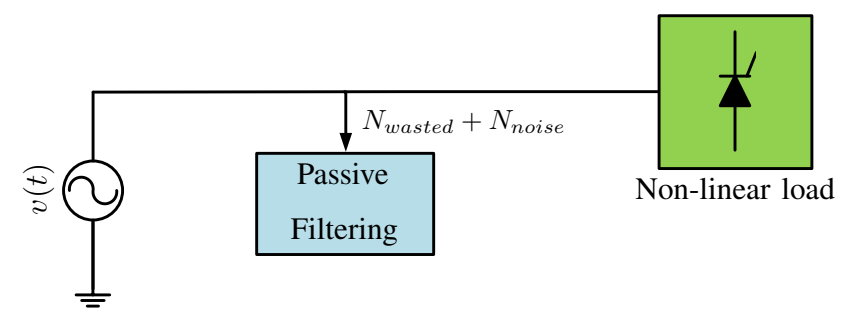

Fig. 2: The Passive Filtering.

element composed of a capacitor and an inductor tuned in a harmonic frequency (e.g., 300 Hertz). This approach results in an electronic device that consumes the energy defined as $P_{\text {wasted }}+P_{\text {noise }}$. A description of such PFER device can be found in [7], where the 5th harmonic component was considered to illustrate the energy recovery approach through a harmonic content reduction scheme.

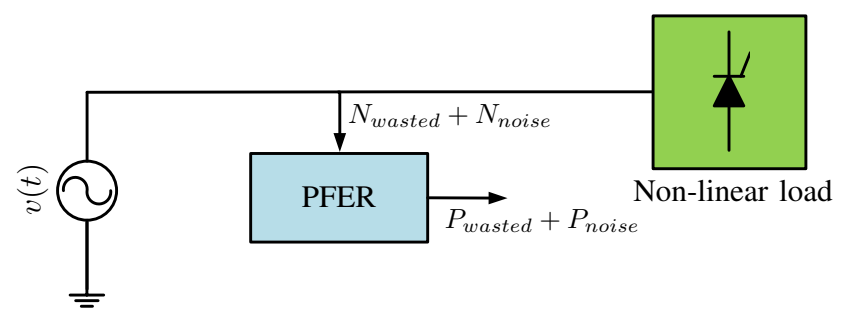

Fig. 3: The Passive Filtering Energy Recovery.

\section{NumericAl ANALYSES}

Numerical results are considered to illustrate the benefits of using an energy recovery in the undesirable components of electric signals. The data set was acquired in a measurement campaign held in the Electrical Engineering Post graduation building at Federal University of Juiz de Fora, Minas Gerais. In our analyses we used only the harmonic range from 2 nd up to 11th component, considering the most of the measured active power is from harmonic components produced by AC/DC converters. Moreover, acquisitions were made in a 10 minutes interval and performed for two weeks straight, covering voltage and current signals in the three phases (A, $\mathrm{B}$, and $\mathrm{C}$ ). The instrument used in the measurement campaign was the Fluke 435-II Power Quality and Energy Analyzer.

Our analysis was done considering only harmonic components so we rely on (7), by excluding the fundamental frequency over $N_{A}$ and taking its real part, since the active power is can be extracted in reality. Firstly, we illustrate the ratio of the so-called $P_{\text {trash }}$, which corresponds to the active power wasted in undesirable components in electric signals, and $P_{\text {useful }}$, which is associated with the active power of the fundamental frequency. The ratio $P_{\text {trash }} / P_{\text {useful shows us how }}$ large is the amount of wasted energy in comparison to what is really being used by an electrical load. It can be seen that the power ratio profile vary from $0.32 \%$ up to $0.48 \%$ and is relatively similar for both weeks.

Moreover, we present an economic analysis considering the Brazilian electricity marked as a case of study. With this in mind we used data available online at Brazilian Electricity 

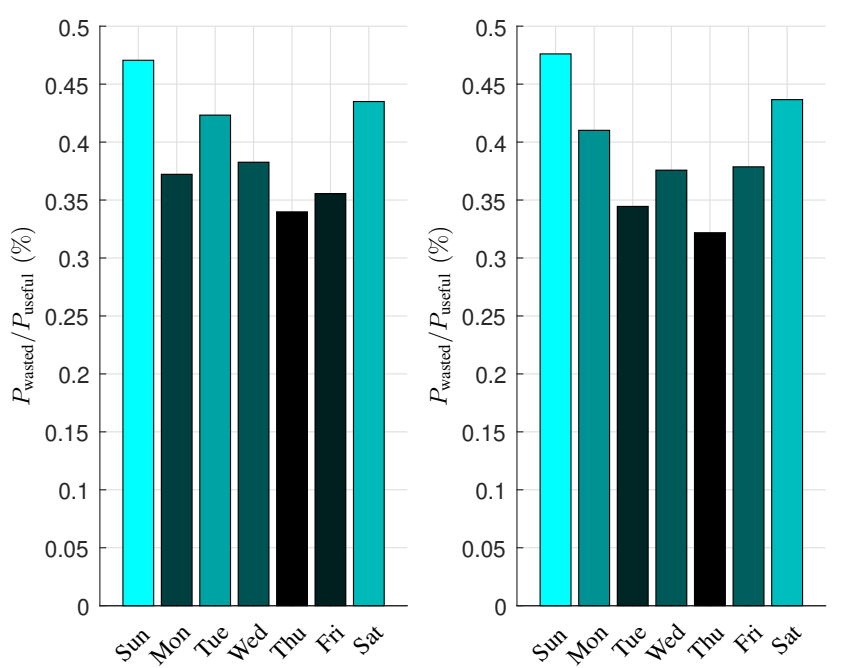

Fig. 4: Power ratio for distinct days of the week in Week \#1 (left) and Week \#2 (right).

Regulatory Agency (ANEEL) [9], which allowed us to calculate rough estimates of wasted energy (in millions of US\$ dollars) in Brazil in 2019, considering the absence of an energy recovery process. In other words, the energy efficiency factor $(\eta)$ is equal to zero. It corresponds to economic losses of US\$ 157.64 million in Brazil in 2019. Note that the averages of estimated economic losses vary from US\$ 90 thousand up to US\$ 78.21 million, depending on the electricity sector. As can be seen, the highest energy losses in 2019 were in the residential, commercial and industrial sectors, in this order.

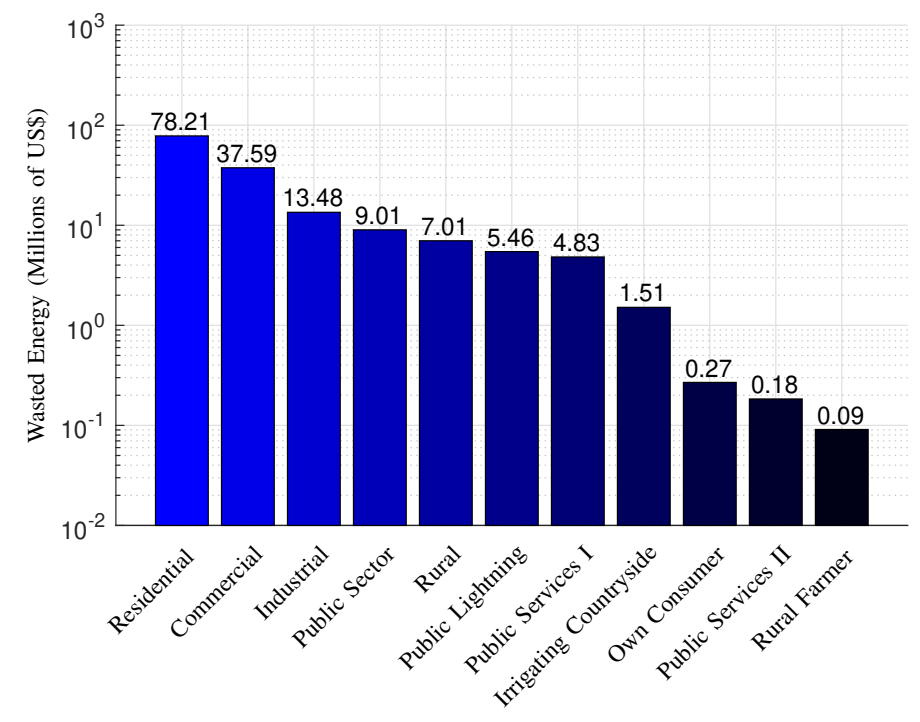

Fig. 5: Average rough estimates of economic losses of different sectors of the Brazilian electricity market with the wasted energy associated with undesirable components in electric signals in 2019 $(\eta=0)$.

Finally, 6 illustrates rough estimates of Brazilian economic losses when an energy recovery process is applied to the Brazilian electricity sectors in 2019. $\eta=0$ informs that savings between 132.20 and 189.15 million dollars can be obtained by a complete recovery of $P_{\text {wasted }}$, which means $\eta$

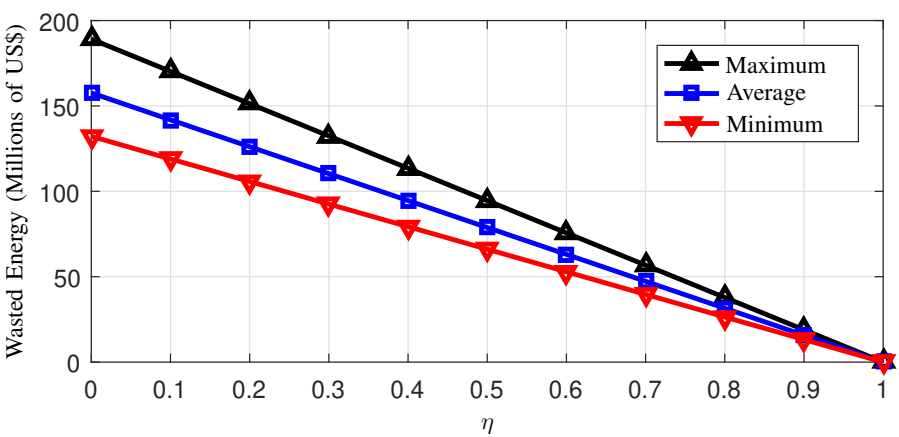

Fig. 6: Rough estimates of Brazilian economic losses (millions of US\$) as functions of $\eta$ in the year 2019 due to the wasted energy with harmonic components.

goes to 1 . In other words, $\eta=0$ means that no filtering process is being considered and $\eta=1$ that an energy recovery approach (e.g., PFER) is performed to decrease energy losses up to zero. It is also worth mentioning that $\eta \rightarrow 1$ can only be accomplished in theory. For illustrative purposes, some values of the rough estimates of economic losses, in millions of dollars, for typical values of $\eta$ are listed in Table I. Based on the fact that $\eta \in[1 / 2,3 / 4]$ results in feasible solutions, savings between $50 \%$ and $75 \%$ of economic losses can be attained, thus illustrating the benefits of the PFER to the electricity sector.

TABLE I: Rough estimates of Brazilian economic losses (millions of US\$) for a finite set of $\eta$ values in the year 2019 .

\begin{tabular}{cccc}
\hline$\eta$ & Min. & Average & Max. \\
\hline 0 & 132.20 & 157.64 & 189.15 \\
\hline $1 / 4$ & 99.15 & 118.23 & 141.86 \\
\hline $1 / 2$ & 66.10 & 78.82 & 94.57 \\
\hline $3 / 4$ & 33.05 & 39.41 & 47.29 \\
\hline
\end{tabular}

\section{CONCLUSION}

This paper presented the use of the waste-to-energy concept in electric power systems, focusing on executing an energy recovery process in undesirable components in electric signals. Also, we described a mathematical formulation that can be used to quantify the wasted energy in such components. Also, we proposed the Passive Filtering Energy Recovery, which can be performed through a Passive Filtering Energy Recovery device, a low-energy device in series with a Passive Filter, constituting a promising alternative to the energy efficiency field. Also, we presented enhancements to the electric system in terms of energy efficiency and PQ. Lasty, we pointed out that remarkable cost savings can be performed with this approach, thus being a relevant field of study for future research works, mainly to supply energy to low-power consumption devices (e.g., IoT devices).

\section{REFERENCES}

[1] Jo Van Caneghem et al. "Waste-to-energy is compatible and complementary with recycling in the circular economy". In: Clean Technologies and Environmental Policy 21 (Mar. 2019), pp. 925-939. 
[2] W. J. McDonald and H. N. Hickok. "Energy Losses in Electrical Power Systems". In: IEEE Transactions on Industry Applications IA-21.3 (May 1985), pp. 803-819.

[3] C. A. Dortolina and R. Nadira. "The loss that is unknown is no loss at all: a top-down/bottom-up approach for estimating distribution losses". In: IEEE Transactions on Power Systems 20.2 (May 2005), pp. 1119-1125.

[4] V. Farahani et al. "Energy Loss Reduction by Conductor Replacement and Capacitor Placement in Distribution Systems". In: IEEE Transactions on Power Systems 28.3 (Aug. 2013), pp. 2077-2085.

[5] H. Sadeghi Gougheri and M. Kiani. "An inductive voltage-/current-Mode integrated power management with seamless mode transition and energy recycling". In: IEEE Journal of Solid-State Circuits 54.3 (Mar. 2019), pp. 874-884.

[6] V. Fernandes, H. V. Poor, and M. V. Ribeiro. "Dedicated energy harvesting in concatenated hybrid PLC-wireless systems". In: IEEE Transactions on Wireless Communications 19.6 (June 2020), pp. 3839-3853.

[7] M. V. Ribeiro et al. "Benefits of energy recovery from the undesirable components of electric signals in electric power systems". In: International Journal of Electrical Power and Energy Systems (2021). (in press).

[8] M. V. Ribeiro and J. L. R. Pereira. "Classification of single and multiple disturbances in electric signals". In: EURASIP Journal on Advances in Signal Processing 2007.056918 (Dec. 2007), pp. 1-19.

[9] ANEEL. Electric energy consumption and revenue reports. Www . aneel . gov . br/relatorios - deconsumo-e-receita. Accessed: June 27, 2020. 